\title{
Cluster observations of structures at quasi-parallel bow shocks
}

\author{
E. A. Lucek ${ }^{1}$, T. S. Horbury ${ }^{1}$, A. Balogh ${ }^{1}$, I. Dandouras ${ }^{2}$, and H. Rème ${ }^{2}$ \\ ${ }^{1}$ Space and Atmospheric Physics, The Blackett Laboratory, Imperial College, London, UK \\ ${ }^{2}$ CESR, BP 4346, 31028 Toulouse Cedex 4, France
}

Received: 10 September 2003 - Revised: 20 January 2004 - Accepted: 23 January 2004 - Published: 14 July 2004

Part of Special Issue "Spatio-temporal analysis and multipoint measurements in space"

\begin{abstract}
Collisionless quasi-parallel shocks are thought to be composed of a patchwork of short, large-amplitude magnetic structures (SLAMS) which act to thermalise the plasma, giving rise to a spatially extended and time varying shock transition. With the launch of Cluster, new observations of the three-dimensional shape and size of shock structures are available. In this paper we present SLAMS observations made when the Cluster tetrahedron scale size was $\sim 100 \mathrm{~km}$. The SLAMS magnetic field enhancement is typically well correlated between spacecraft on this scale, although small differences are observed. The statistical characteristics of these differences contain information on the typical gradients of magnetic field changes within the SLAM structure which, in the case studied here, occur on scales of $100-150 \mathrm{~km}$, comparable with the upstream ion inertial length.
\end{abstract}

Key words. Interplanetary physics (planetary bow shocks; plasma waves and turbulence)

\section{Introduction}

The observed magnetic field and plasma signatures of a collisionless shock transition depend on both the ambient plasma conditions, and on the angle between the magnetic field and the normal to the shock surface, $\theta_{B N}$. When the magnetic field is nearly parallel to the normal: a quasi-parallel shock, particles are able to escape into the region upstream of the shock where they generate and scatter from waves (e.g. Le and Russell, 1992). Under these conditions the shock transition tends to be extended and unsteady (e.g. Greenstadt et al., 1982). Embedded in the large variations in magnetic field magnitude and direction typically associated with this type of shock are discrete structures which have been called short, large-amplitude magnetic structures (SLAMS). These have a relatively smooth magnetic field signature, where the magnetic field magnitude $|B|$ is enhanced over the background

Correspondence to: E. A. Lucek

(e.lucek@ic.ac.uk) by a factor of 2 or more. SLAMS occur both within relatively undisturbed upstream plasma (isolated SLAMS) and in partially thermalised plasma (embedded SLAMS). They propagate sunwards in the plasma frame, although they are typically convected anti-sunwards in the plasma flow (Thomsen et al., 1990; Schwartz et al., 1992). Study of SLAMS (Thomsen et al., 1990; Schwartz and Burgess, 1991; Schwartz et al., 1992; Mann et al., 1994), together with the results of simulation work (e.g. Scholer, 1990; Thomas et al., 1990; Scholer, 1993; Giacalone et al., 1994; Scholer et al., 2003), support a picture of the quasi-parallel shock transition as a time varying, spatially extended and inhomogeneous region composed of a patchwork of SLAM structures which grow, steepen, and act as a new shock front (e.g. Burgess, 1989).

Simulation studies of the propagation of ultra-low frequency (ULF) waves into a supra-thermal particle pressure gradient (Dubouloz and Scholer, 1995) showed that under these conditions a wave grew into a nonlinear structure with enhanced magnetic field magnitude, similar to a SLAM structure. Simulations also showed that SLAMS-like features could grow very rapidly from ULF waves, on the time scale of seconds (Giacalone et al., 1994). Further analysis of SLAMS showed that they were associated with a gradient in supra-thermal particle pressure, consistent with theory and the simulation results (Giacalone et al., 1993).

The scale size and shape of SLAMS structures is relevant to the understanding of how they interact with the plasma, but until recently SLAMS observations have been restricted to those made by single and dual satellite missions. The SLAMS identified by Schwartz and Burgess (1991) had a scale size in the flow direction of approximately $0.5 R_{E}$, and the authors proposed that the flow transverse SLAMS scale might be of a comparable scale size to that of the ULF waves from which they grow. Le and Russell (1990) used dual spacecraft studies of the correlation length of ULF foreshock waves to infer a flow perpendicular correlation length of $\sim 1 R_{E}$. Simulations, however, also showed that SLAMSlike structures not only grew as they were convected back towards the shock surface, but that they were also refracted and became filamentary in nature (Dubouloz and Scholer, 1995). 
In 2000 the four Cluster spacecraft were launched into a polar orbit with apogee at $19.6 R_{E}$. The spacecraft fly in a tetrahedron configuration, allowing simultaneous measurements of the plasma in three independent directions for the first time. Comparison of the data recorded by the four spacecraft gives information about the plasma on approximately the scale size of the tetrahedron, which is altered over the course of the mission, in order study plasma processes on a range of scales from $\sim 100$ to $10000 \mathrm{~km}$.

An earlier paper described the first Cluster observations of SLAMS when the tetrahedron scale was $\sim 600 \mathrm{~km}$ (Lucek et al., 2002). This study was limited by the observation of only a few well developed quasi-parallel shocks, but analysis of a small number of SLAMS-like features showed that although all four spacecraft typically encountered each structure, significant differences were seen in the magnetic field profiles at the four spacecraft. In these cases the differences were not consistent with the growth of the SLAMS as they crossed the satellite formation. The observations were, however, consistent with the overall SLAMS size, exceeding that of the tetrahedron but, unexpectedly, with significant gradients in the magnetic field being present on the spacecraft separation scale of $\sim 600 \mathrm{~km}$. It was suggested that these differences could either arise from the spacecraft sampling the gradient at the edges of the SLAMS, or from SLAMS having internal "lumpy" structure. The presence of these differences made it difficult to estimate the propagation direction of the SLAMS.

In 2002, when the tetrahedron scale was approximately $100 \mathrm{~km}$, Cluster encountered several well developed quasiparallel shocks. In this paper we show several examples of SLAMS-like features. We present one example in some detail and then use an interval containing multiple SLAMS to address what can be learnt about the scale size of these structures transverse to the flow direction. A detailed statistical study of SLAMS, which is required to draw general conclusions on their 3-dimensional properties, is currently underway.

\section{Analysis}

In this paper we present data from two quasi-parallel shock transitions: 3 February 2002 (day 34), 04:00-07:00 UT, and 20 February 2002 (day 51), 15:30-17:30 UT. Both were observed when the Cluster tetrahedron scale was $\sim 100 \mathrm{~km}$. The first crossing was characterised by unusually high ULF wave activity in the foreshock. Unlike the majority of ULF waves, those on this day were left-handed in the spacecraft frame (Eastwood et al., 2003) and hence right-handed in the plasma frame. The wave activity was persistent on this day and was interspersed with intervals containing SLAMS, making this a good example to study when comparing SLAMS and ULF wave properties, and such a study is ongoing. The second crossing contained a number of well developed, isolated SLAM structures, one of which is presented in the next section.

\subsection{Example of an isolated SLAMS}

Figure 1 shows a well developed SLAM-like structure observed on 20 February 2002. At this time the Cluster tetrahedron scale was $\sim 100 \mathrm{~km}$, and was located at $\sim 14 \mathrm{LT}$, about $8.3 R_{E}$ above the ecliptic plane. Using the ACE magnetic field orientation, with a time lag chosen to match up recognisable features in the ACE and Cluster data sets, the predicted $\theta_{B N}$ at the location of Cluster (not shown), relative to a model shock normal (Peredo et al., 1995) was consistently below $45^{\circ}$ throughout the crossing, in keeping with Cluster encountering a quasi-parallel shock transition.

Figure 1 shows magnetic field data from the FGM instruments (Balogh et al., 2001) in the top six panels, with the proton number density $\left(N_{P}\right)$ and plasma speed $(|V|)$ measured by the CIS instruments (Rème et al., 2001) in the bottom two panels. The SLAMS-like magnetic field enhancement started just before 17:07:15, lasted for $\sim 6 \mathrm{~s}$, and had a very similar profile at all four spacecraft, with significantly higher correlations between the spacecraft than was observed when the tetrahedron scale was $\sim 600 \mathrm{~km}$. $|B|$ peaked at a value 3.5 times the background magnetic field value, and the structure contained a clear rotation of the magnetic field vector, with mixed left- and right-handed polarisation sense. The order in which the spacecraft encountered the structure is related to its orientation and velocity relative to the plasma flow. By assuming that the structure was planar on the tetrahedron scale, and that it was moving with constant velocity, the relative timings can be used to determine a normal defining the SLAMS orientation, and a velocity along this normal. In this case the timings implied that the SLAMS was convected anti-sunward over the tetrahedron, but that the SLAMS was moving sunwards in the plasma frame, and hence the magnitude of the SLAMS spacecraft frame velocity was lower than the background plasma velocity. The exit edge, which was therefore the leading edge of the SLAMS in the plasma frame, had a clear high frequency wave associated with it. This wave was left-hand polarised in the spacecraft frame, consistent with a whistler wave propagating against the solar wind flow. These characteristics are all consistent with those of previously identified SLAMS (e.g. Schwartz et al., 1992; Mann et al., 1994).

The timing of the spacecraft as they entered the SLAMS was different from that when they exited: this can be seen most clearly in the comparison of Cluster 3 (green) with the other spacecraft. Comparison of the entry and exit showed that the spacecraft signatures were consistent with an expansion of the SLAMS structure under internal pressure, but that they could also have arisen from the SLAMS having significant curvature on the spatial scale of the tetrahedron, i.e. the change in time delay represents the three-dimensional structure of the SLAMS.

Before the spacecraft encountered the SLAM structure they passed through a region which appeared to contain partially thermalised plasma, with enhanced proton number density and decreased plasma velocity. The magnetic field measured by the four Cluster satellites in this region showed a 


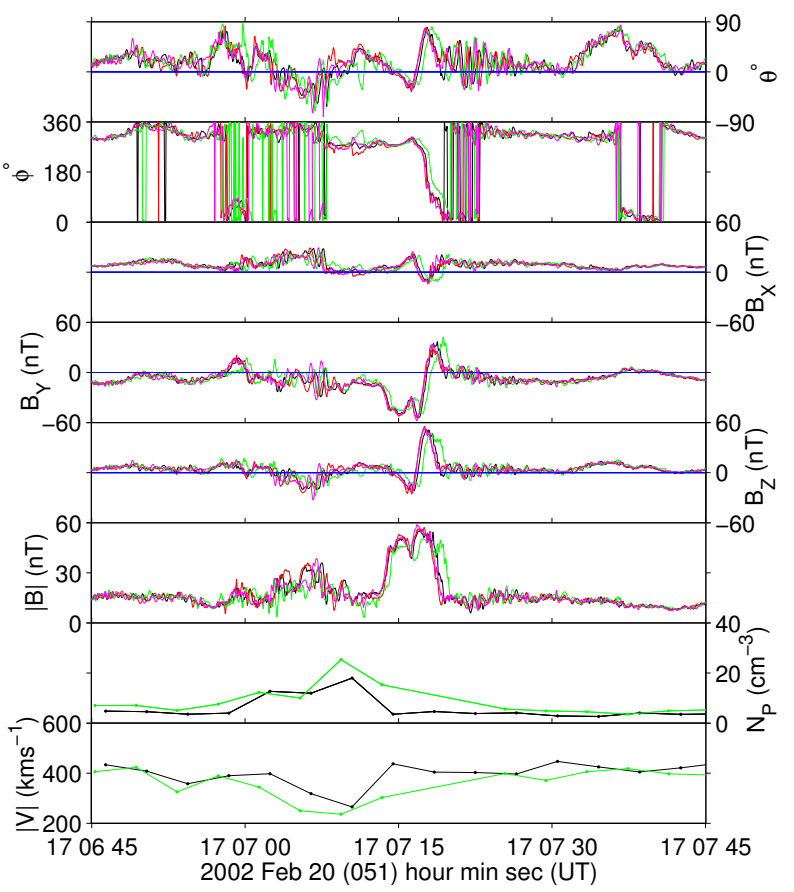

Fig. 1. An example of a well developed SLAM structure. Panels show magnetic field elevation and longitude angles, $\theta$ and $\phi$ (degrees), $B_{X}, B_{Y}, B_{Z}$ in GSE co-ordinates, magnetic field magnitude $|B|(\mathrm{nT})$, proton number density $N_{P}\left(\mathrm{~cm}^{-3}\right)$, and plasma flow speed $|V|(\mathrm{km} / \mathrm{s})$. The different colours show data from the four spacecraft: 1 (black), 2 (red), 3 (green) and 4 (magenta).

lower level of correlation, suggesting that significant variations in the plasma occurred on scales of $100 \mathrm{~km}$ in this region. Such small-scale processes might be related to plasma thermalisation.

\subsection{Signatures of SLAMS scale size}

Another quasi-parallel shock occurred on 3 February 2002 while Cluster was located near noon, $\sim 8.3 R_{E}$ below the ecliptic plane. The region upstream of the shock was populated by exceptionally well developed ULF waves, visible most clearly in the magnetic field elevation and longitude angles (Fig. 2), and by SLAMS, visible as increases in $|B|$. When the tetrahedron scale was $\sim 100 \mathrm{~km}$, the time delay between the different spacecraft observing an enhancement in magnetic field magnitude was significantly shorter than the duration of the SLAMS. The shape of the magnetic field enhancement was also generally similar at these scales, although the peak amplitude typically showed small differences between spacecraft. Therefore, we are confident that at this tetrahedron scale we identify events which were sampled by all four spacecraft. Since this interval contained multiple SLAMS, small differences between pairs of spacecraft can be analysed statistically: these differences contain information on the gradients at SLAMS, and on their overall shape and size.

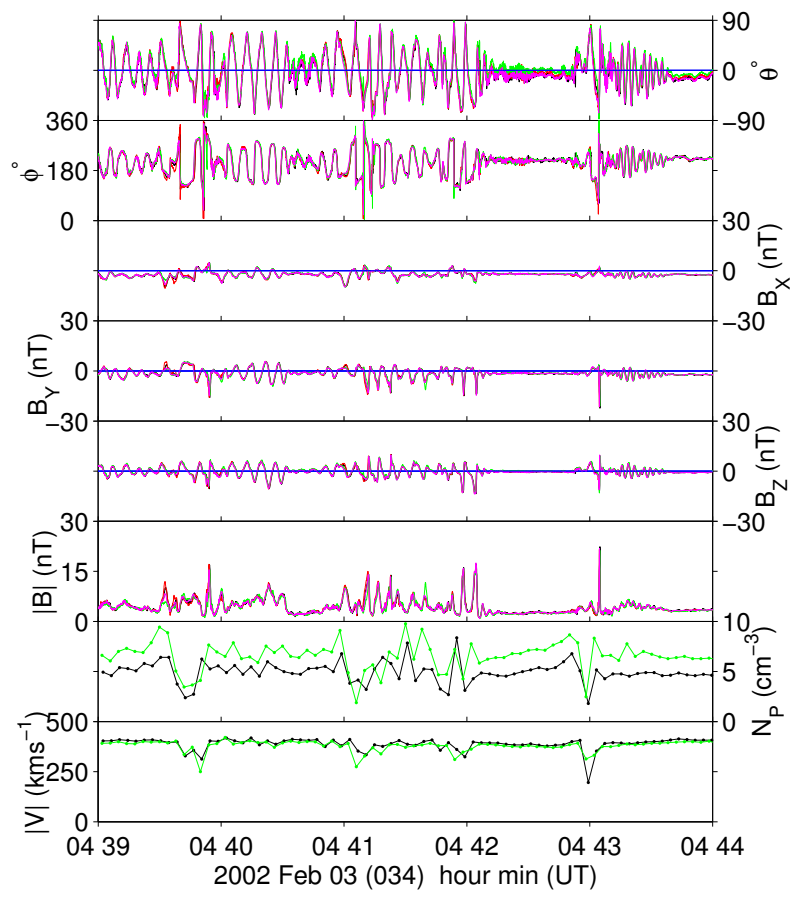

Fig. 2. Part of an interval containing both well developed ULF waves and SLAMS. Panels show the same information as in Fig. 1.

Between $\sim$ 04:00 and 05:30 UT 28 SLAMS were identified. The duration of each event was estimated from the time for which the magnetic field magnitude exceeded half the maximum $|B|$ observed within the event. This method underestimated the total duration of the event, but avoided the analysis being influenced by high frequency whistler waves which can occur at either the leading or trailing edges of the SLAMS. The integrated flux at each spacecraft was estimated by integrating $|B|$ through each event. The differences in total flux between the different pairs of spacecraft, relative to the maximum observed, were calculated. These differences were then plotted as a function of the separation of the spacecraft pair perpendicular to the solar wind velocity vector, i.e. transverse to the plasma flow, in order to look for evidence of a spatial gradient in the magnetic field signature. Also of interest is whether the SLAMS show significant time evolution between the spacecraft. We might expect to see evidence for this effect more clearly in the distribution of differences ordered by spacecraft separation along the plasma flow vector, since this determines the time delay between the same structure crossing the different satellites. We have found some evidence for time evolution, and these results will be presented in a later publication.

When the differences are plotted against the spacecraft separation perpendicular to the solar wind flow vector, they form a triangular shaped scatter plot (Fig. 3). Therefore, when the spacecraft have a small separation perpendicular to the solar wind flow vector the probability of two spacecraft observing large differences in SLAMS signature was much lower than when the spacecraft were more widely separated. 


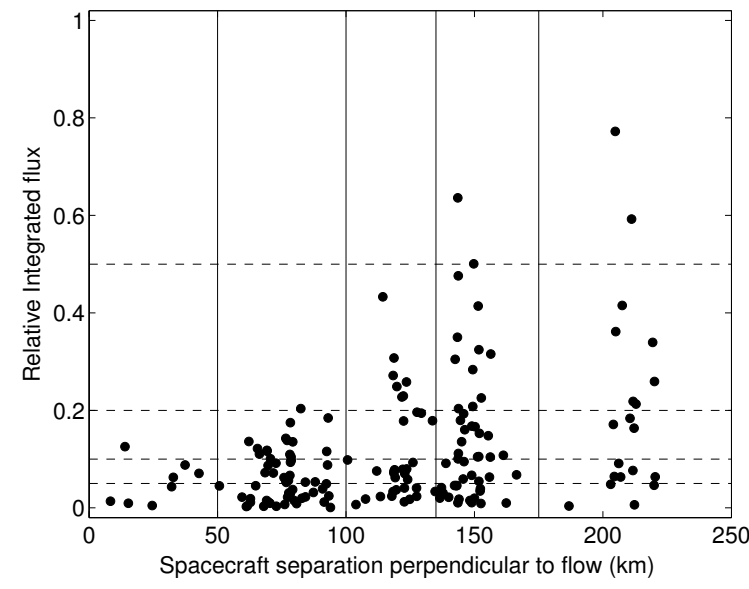

Fig. 3. Differences in SLAMS' integrated flux measured by the different Cluster pairs, normalised by the maximum value observed at each event, plotted as a function of the separation of the two satellites perpendicular to the flow direction. Vertical lines indicate the division of the points by spacecraft separation transverse to the plasma flow used to generate Fig. 4. Horizontal dashed lines indicate the thresholds used to characterise the distribution of differences between spacecraft.

The lack of a large population of points exceeding 0.5 , even for the largest separations of $\sim 250 \mathrm{~km}$, suggests that the overall extent of the SLAMS perpendicular to the solar wind flow was greater than the tetrahedron scale, consistent with previous Cluster observations.

The solid vertical lines in Fig. 3 divide the points into 5 bins in spacecraft separation perpendicular to the solar wind velocity vector. These values were chosen to give a fair sample of points within each separation range. The dashed horizontal lines indicate four thresholds in observed difference between a pair of spacecraft. The scatter distribution can be crudely characterised by counting the fraction of points in each vertical bin which exceed each difference threshold. These fractions are plotted in Fig. 4.

The distributions in Fig. 4 show that for about half of the time two spacecraft separated by only $25 \mathrm{~km}$ observed a difference in SLAMS' normalised flux exceeding 0.05. This fraction increased with increasing distance between spacecraft, as expected. In contrast, the likelihood of two spacecraft at $25 \mathrm{~km}$ separation observing a large difference, such as 0.5 , was zero within this small sample. Large differences were not observed unless the spacecraft were separated across the solar wind flow by more than $150 \mathrm{~km}$.

The distribution of differences contains information about the shape, size and gradients of SLAMS, but a larger sample of data is required to characterise these properties more completely. For example, with only a small number of events we are not able to investigate the time variation of the gradient scale, which might be expected to evolve as the SLAMS are convected towards the shock. Also, using data from a single shock means that we cannot consider the dependence of the gradient scale on the background plasma conditions

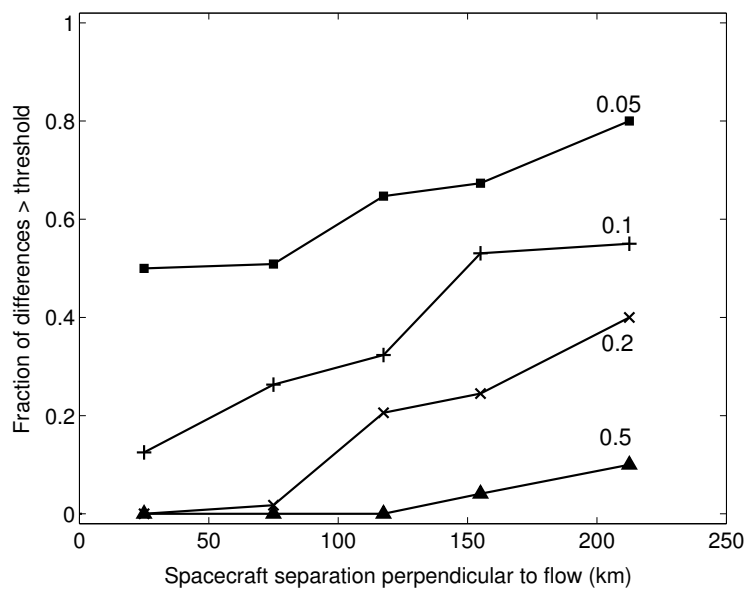

Fig. 4. The change in occurrence of observed differences between spacecraft as a function of spacecraft separation perpendicular to the flow. Each trace shows the fraction of observed differences between pairs of spacecraft which exceed a certain threshold: $0.05,0.1,0.2$, and 0.5 , each plotted as a function of flow transverse separation.

or variations in shock orientation. The distributions could also usefully be compared with simulations of SLAMS-like structures.

\section{Summary}

Cluster observations of SLAMS within quasi-parallel shocks have now been made at scales of 100 and $600 \mathrm{~km}$. Earlier observations at scales of $\sim 600 \mathrm{~km}$ showed that although the overall SLAMS extent exceeded $600 \mathrm{~km}$, significant differences were observed between spacecraft at these separations. Shocks observed at scales of $100 \mathrm{~km}$ now show that SLAMS are much better correlated on these scales, although regions with smaller scale variations downstream of the SLAMS (in the plasma frame) might be associated with thermalisation processes. Statistical analysis of the small differences in $|B|$ measured by multiple spacecraft suggest that while the SLAMS extent is significantly greater than $100 \mathrm{~km}$, as expected, the gradient scale in $|B|$ appears to be of the order of $100-150 \mathrm{~km}$, which is comparable with the ion inertial length in the undisturbed upstream solar wind at this time.

Acknowledgements. Cluster data analysis at Imperial College is supported by PPARC, E. A. Lucek and T. S. Horbury are supported by PPARC Fellowships. Cluster CIS analysis at CESR is supported by a CNES grant. The authors would like to thank Prof S. J. Schwartz for helpful discussions about shocks and SLAMS. The authors are also grateful to the two referees for their constructive comments.

Topical Editor T. Pulkkinen thanks two referees for their help in evaluating this paper. 


\section{References}

Balogh, A., Carr, C. M., Acuña, M. H., Dunlop, M. W., Beek, T. J., Brown, P., Fornacon, K.-H., Georgescu, E., Glassmeier, K.H., Harris, J., Musmann, G., Oddy, T., and Schwingenschuh, K.: The Cluster Magnetic Field Investigation: overview of in-flight performance and initial results, Ann. Geophys., 19, 1207-1217, 2001

Burgess, D.: Cyclic behaviour at quasi-parallel collisionless shocks, Geophys. Res. Lett., 16, 345-348, 1989.

Dubouloz, N. and Scholer, M.: Two-dimensional simulations of magnetic pulsations upstream of the Earth's bow shock, J. Geophys. Res., 100, 9461-9474, 1995.

Eastwood, J. P., Balogh, A., Lucek, E. A., Mazelle, C., and Dandouras, I.: On the existence of Alfvén waves in the terrestrial foreshock, Ann. Geophys., 21, 1457-1465, 2003.

Giacalone, J., Schwartz, S. J., and Burgess, D.: Observations of suprathermal ions in association with SLAMS, Geophys. Res. Lett., 20, 149-152, 1993.

Giacalone, J., Schwartz, S. J., and Burgess, D.: Artificial spacecraft in hybrid simulations of the quasi-parallel Earth's bow shock: analysis of time series versus spatial profiles and a separation strategy for Cluster, Ann. Geophys., 12, 591-601, 1994.

Greenstadt, E. W., Hoppe, M. M., and Russell, C. T.: Largeamplitude magnetic variations in quasi-parallel shocks: correlation lengths measured by ISEE 1 and 2, Geophys. Res. Lett., 9, 781-784, 1982.

Le, G. and Russell, C. T.: A study of the coherence length of ULF waves in the Earth's foreshock, J. Geophys. Res., 95, 10703 $10706,1990$.

Le, G. and Russell, C. T.: A study of ULF wave foreshock morphology - II: Spatial variation of ULF waves, Planet. Space Sci., 40, 1215-1225, 1992.

Lucek, E. A., Horbury, T. S., Dunlop, M. W., Cargill, P. J., Schwartz, S. J., Balogh, A., Brown, P., Carr, C., Fornacon, K.H., and Georgescu, E.: Cluster magnetic field observations at a quasi-parallel bow shock, Ann. Geophys., 20, 1699-1710, 2002.
Mann, G., Lühr, H., and Baumjohann, W.: Statistical analysis of short large-amplitude magnetic field structures in the vicinity of the quasi-parallel bow shock, J. Geophys. Res., 99, 13315 $13323,1994$.

Peredo, M., Slavin, J. A., Mazur, E., and Curtis, S. A.: Threedimensional position and shape of the bow shock and their variation with Alfvénic, sonic and magnetosonic Mach numbers and interplanetary field orientation, J. Geophys. Res., 100, 79087916, 1995.

Rème, H., Aoutstin, C., Bosqued, J.-M., et al.: First multispacecraft ion measurements in and near the Earth's magnetosphere with the identical Cluster ion spectrometry (CIS) experiment, Ann. Geophys., 19, 1303-1354, 2001.

Scholer, M.: Diffuse ions at a quasi-parallel collisionless shock: simulations, Geophys. Res. Lett., 17, 1821-1824, 1990.

Scholer, M.: Upstream waves, shocklets, short large-amplitude magnetic structures and the cyclic behaviour of oblique quasiparallel collisionless shocks, J. Geophys. Res., 98, 47-57, 1993.

Scholer, M., Kucharek, H., and Shinohara, I.: Short large-amplitude magnetic structures and whistler wave precursors in a fullparticle quasi-parallel shock simulation, J. Geophys. Res., 108, 1273-1281, doi:10.1029/2002JA009820, 2003.

Schwartz, S. J. and Burgess, D.: Quasi-parallel shocks: a patchwork of three-dimensional structures, Geophys. Res. Lett., 18, 373376, 1991.

Schwartz, S. J., Burgess, D., Wilkinson, W. P., Kessel, R. L., Dunlop, M., and Lühr, H.: Observations of short large-amplitude magnetic structures at a quasi-parallel shock, J. Geophys. Res., 97, 4209-4227, 1992.

Thomas, V. A., Winske, D., and Omidi, N.: Re-forming supercritical quasi-parallel shocks 1., One- and two-dimensional simulations, J. Geophys. Res., 95, 18 809-18 819, 1990.

Thomsen, M. F., Gosling, J. T., Bame, S. J., and Russell, C. T.: Magnetic pulsations at the quasi-parallel shock, J. Geophys. Res., 95, 957-966, 1990. 